\title{
CFD SIMULATION OF SOLAR LOADING IN CAR
}

\section{RAVI. D}

Associate Professor, Department of Mechanical Engineering, Bharath University,

Chennai, Tamil Nadu, India

\begin{abstract}
A frequent problem faced by the car drivers in Chennai is, the hot suffocating inner of the car following hours of steady solar radiation when parked in an un-shaded area. The heat build-up in the cabin due to solar radiation can increase up to $80^{\circ} \mathrm{C}$ usual. The undesired increase in cabin temperature will affect the human comfort and interior aesthetics of the cabin, such situation can affect the life span of the components in the passenger cabin adversely. Thermal comfort of the occupants in a vehicle cabin is a growing concern due to occupant's health and safety. To bring down the hot cabin temperature to ambient temperature, the driver has to work the air-conditioning system in full load, thus consuming excess fuel. The concept helps in reducing the usage of air conditioner unit to reduce the interior temperature and thus the usage of fuel \& power consumption. Thermal comfort of passengers in an automotive cabin is a challenging and mandatory requirement in today's world, as they have an accountable role on passenger safety and pleasurable drive. Thermal energy storage via phase change materials is one of the most promising candidates as zero power usage method to maintain ambient temperature profile during both the winter and summer seasons. Phase Change Materials are substances that use latent heat energy for the varying of phase from solid to liquid or vice versa. The phase change procedure of a PCM from solid to liquid and liquid to solid occurs at nearly steady temperature. The phase change process of the PCM is directly proportional to the solar load.

KEYWORDS: CFD, Solar Loading, Solar Radiation, Phase Change Materials, Heat Energy \& Temperature
\end{abstract}

Received: Feb 28, 2019; Accepted: Mar 19, 2019; Published: Apr 16, 2019; Paper Id.: IJMPERDJUN201926

\section{INTRODUCTION}

Experimental and computational analyses have been conducted to evaluate the thermal performance of the new design and the normal roofing with different colors. A general mathematic equation system has been derived for the thermal process through the roof. The results demonstrate that the new design could successfully reduce the downward heat flow from the roof into the cabin. As an effect, the cooling load of the cabin could be considerably lower. In this article, a new design of roofing for vehicles has been done by inserting a layer of PCM to the roof structure in order to slow down the heat transfer process through the roof to the cabin space. Both experimental and computational results have shown that the new design has an enhanced thermal performance than the normal roof structure of the accessible vehicles. In the mentioned experiment condition, the new design may help to decrease up to about $40 \%$ of the energy amount necessary for cooling down the heat entering into the cabin from the roof. The saving rate could be even much higher in case external wind is present due to natural wind or movement of the car in use. The paper also suggests conditions for choosing suitable PCM type in real design, according to the weather condition such as ambient temperature, melting temperature and latent heat capacity. This is a hopeful design which could be especially produced due to its simple structure and rational cost. 


\section{LITERATURE SURVEY}

Nguyen $\mathrm{Vu}$ Lan et al (2012), describes that heat flow from the roof mutually with radiation through glass windows obviously contributes in the total heat gained of a vehicle cabin. The contribution is more significant especially hot and sunny weather with little wind. This paper shows a new design for vehicle roofing structure in order to progress its total thermal resistance. Its main concept is to utilize phase change material property to first trap the heat from solar radiation and then discharge it back to the environment by means of the naturally favored external convection when the vehicle is in use or during the nocturnal cycle.

\section{PHYSICS CONDITIONS FOR PCM}

The PCM layer absorbs the heat from the cabin and the adjacent layers for its phase change process. It utilizes the heat in the cabin as sensible heat. The PCM has to be analyzed for solid-liquid and liquid-solid. Eulerian Multiphase model is used to capture the phase changing properties of the material.

The physics conditions for the PCM layer are,

- Energy Source Option

- Initial Condition Option

- Momentum Source Option

- Phase Source Option

The conditions given to the interface below the fan slot to create a condition of forced convection in the cabin are,

- Pressure variation is assumed as $100 \mathrm{~Pa}$, which is atmospheric pressure.

- The rotations per minute of the air flowing through the fan are $250 \mathrm{rpm}$.

\section{ROLE OF COMPUTATIONAL FLUID DYNAMICS}

Computational fluid dynamics, usually abbreviated as CFD, is a branch of fluid mechanics that uses numerical methods and algorithms to solve and analyze problems that involve fluid flows. Computers are used to perform the calculations required to simulate the interaction of liquids and gases with surfaces defined by boundary conditions. With high-speed supercomputers, better solutions can be achieved. Ongoing research yields software that improves the accuracy and speed of complex simulation scenarios such as transonic or turbulent flows. Initial experimental validation of such software is performed using a wind tunnel with the final validation coming in full-scale testing, e.g. flight tests. The application of CFD to simulate cabin climatic conditions has become an integral part of vehicle development process and complements well with wind tunnel tests. However, determining thermal comfort inside the car cabin using CFD requires considering large number of factors to ensure that the simulation results are well in sync with the experimental test values. The thermal analysis of car compartment involves not only the complex geometry, but also a strong interaction between airflow and all the three modes of heat transfer: conduction, convection and radiation. Failing to comprehensively include the details in the CFD model will lead to inaccuracy in results and drastically increase the consumption of computational power. 


\section{ANSA}

ANSA is a computer-aided engineering tool for Finite Element Analysis and CFD Analysis widely used in the automotive industry. It is developed by the BETA CAE Systems S.A., Greece. In the United States, it is distributed by Beta CAE Systems, USA, based in Farmington Hills, Michigan.

ANSA maintains the association between CAD geometry and the FE mesh. This means that the FE meshes are better representations of their geometric parents. Also it is easy to maintain and update any changes in the geometry by simply reworking the updated area instead of recreating the FE from scratch. It carries several proprietary algorithms for meshing suitable for both CFD and structural models. ANSA stands for 'Automatic net generation for structural analysis'.

ANSA has broadly six menus which are used to do various activities those are: TOPO, MESH, VMESH, DECK-SOLVER, MORPH, HBLOCK.

ANSA has been in the forefront of offering top notch pre-processing solutions to various automotive problems. Sophisticated tools like task manager, tank tool, morphing, section analysis, and the optimization tool make it a breeze to build and setup models to meet various safety standards. ANSA's CAD associative automated meshing and advanced design optimization set up tools are helping us set the standards in finite element pre-processing and shortening the design cycle. ANSA is widely used in the industry to build complex models for

- Structural analysis

- Crash analysis

- $\quad \mathrm{NVH}$ analysis

- CFD analysis

- Occupant safety analysis

- Body-in-white, chasis and trimmed body modelling

In regard to this project, ANSA is used to create the effective Surface mesh of the passenger cabin designed. The surface meshing of the cabin is done using the Triangular mesh element so as to satisfy its criteria to understand accurate properties.

\section{Star CCM+}

STAR-CCM+ is unrivalled in its ability to tackle problems involving multi- physics and complex geometries. STAR-CCM+ has an established reputation for producing high-quality results in a single code with minimum user effort. The net result of this is that engineers get to spend more time actually analyzing engineering data and less time preparing and setting up simulations. The first "official release" of STAR-CCM+ included the world's first commercially available polyhedral meshing algorithm. The use of a polyhedral mesh has proven to be more accurate for fluid-flow problems than a hexahedral or tetrahedral mesh of a similar size (number of cells), but is considerably more difficult to create. In this project, Star CCM+ has been effectively used for creating volume mesh of the passenger cabin and to solve the mesh under the given conditions. The post-processing tool provided with the temperature contour report and the graphical representation of the surface temperatures in the roof layers. 


\section{RESULTS AND DISCUSSIONS}

\section{Thermal Variation in Passenger Cabin}

\section{Analysis Result of Car Cabin with Normal Roof}

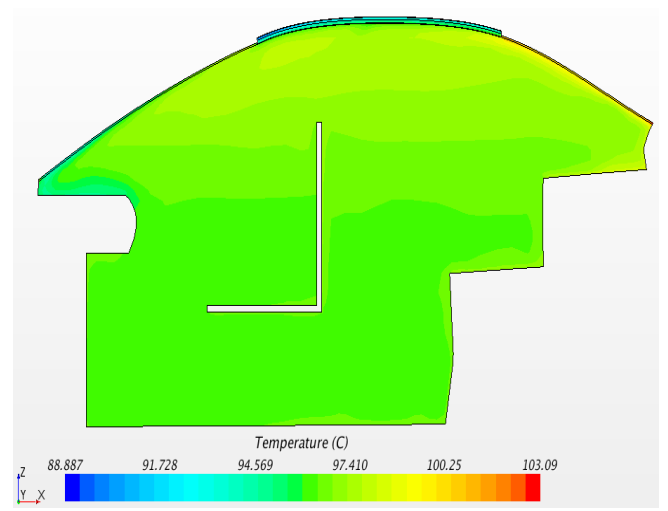

Figure 1

\section{Temperature Contour of Cabin with Normal Roof}

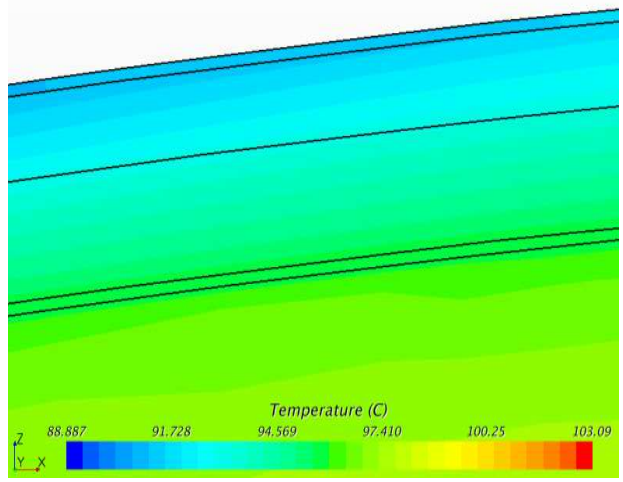

Figure 2

\section{Enlarged View of Normal Roof}

In this case, the car cabin is analyzed for the temperature variation for 1hour when parked in an un-shaded region with normal roof structure.


Figure 3 


\section{CONCLUSIONS}

The boundary conditions, physical models and values are solved by the Star CCM+ solver and the above analysis report has been justified as passenger comfort is based on the interior temperature, the temperature contour of all the different conditions is analyzed.

\section{REFERENCES}

1. Aroussi, A. and Aghil, S., "Characterisation of the Flow Field in a Passenger Car Model " Optical Diagnostics in Engineering, 4(1): 1-15 (2000),.

2. Mann, Martin and Haigis, Matthias, "Numerical Investigation of the Ventilation and Thermal comfort in a Commuter Train ", Arsenal Research, Business Area Transport Technologies, Vienna, Austria.

3. Ambs, Raymond, “Improved Passenger Thermal Comfort Prediction in the Preprototype Phase by Transient Interior CFD Analysis Including Mannequins”, SAE Technical Paper Series, 2002-01-0514, U.S.A.

4. Han, Taeyoung, "Validation of 3-D Passenger Compartment Hot Soak and Cool-Down Analysis for Virtual Thermal Comfort Engineering”, SAE Technical Paper Series, 2002-01-1304, U.S.A.

5. Alexandrov, Alex, Kudriavtsev, Vladimir and Reggio, Marcelo, "Analysis of Flow Patterns and Heat Transfer in Generic Passenger Car Mini-Environment ”, 9th Annual Conference of the CFD Society of Canada, 27-29 May, 2001, Kitchener, Ontario.

6. Kumar, R., Pedgopu, V., Kumar, Anil., Thakur, Robin., \& Pundir, Anil. (2013). CFD based analysis heat transfer and friction characteristics of broken multiple rib roughened solar air heater duct. Int J Mech Prod Eng Res Dev, 3, 165-172.

7. Ideriah, F.J.K., "Prediction of Turbulent Cavity Flow Driven by Buoyancy and Shear”, Journal of Mechanical Engineering Science, 22:287-295 (1984).

8. Awbi, H.B. and Setrak, A.A., "Numerical Solution of Ventilation Air Jet", The Fifth Int. Symposium on the Use of Computer for Environmental Engineering Related to Building, Bath, England (1986).

9. Shinichiro Wakashima: on Development of the Thermal Environment Simulator of an Automobile, 47th Japan Heat Transfer Symposium proceedings (2010).

10. Tomohiro Kitagawa, Kiyoshi Kawaguchi, Daisuke Watanabe and Ryota Toyohara: Reducing Temperature in a Parking Automobile under Summer Solar Radiation, 49th Japan Heat Transfer Symposium proceedings (2012). 
